\title{
Versorgungsforschung und die Patientenperspektive
}

\section{NORBERT SCHMACKE}

Prof. Dr. Norbert Schmacke ist Hochschullehrer für Gesundheitswissenschaften an der Universität Bremen und Mitglied des Instituts für Public Health und Pflegeforschung (IPP). Er ist unparteiisches Mitglied im Gemeinsamen Bundesausschuss

\author{
Es ist Konsens in der wissenschaftlichen Literatur \\ und in der gesundheitspolitischen Debatte, dass \\ die Perspektive von Patienten und Patientinnen \\ für die Analyse bestehender sowie die Entwicklung \\ künftiger Versorgungsmodelle ins Blickfeld zu \\ nehmen ist. Versorgungsforschung ist in besonderer \\ Weise gefordert, diesen hohen Anspruch mittels \\ qualitativer und quantitativer Forschungsmethoden \\ umzusetzen. Dabei gerät leicht aus dem Blickfeld, \\ dass trotz aller Fortschritte immer noch an den \\ Fundamenten einer patientenzentrierten Medizin \\ gearbeitet werden muss. Dies gilt insbesondere \\ für das inzwischen fast umgangssprachlich \\ gewordene "Shared Decision Making".
}

Den Blick von Patienten und Patientinnen auf die individuelle Untersuchungsund Behandlungssituation wie auf die Rahmenbedingungen der Versorgung zum Forschungsthema zu erheben: das sollte eigentlich am Beginn des 21. Jahrhunderts kein besonders aufregendes Thema mehr sein. Und Versorgungsforschung kann sich eigentlich nur deshalb anheischig machen, für das Thema besonders sensibel zu sein, weil sie explizit danach fragt, was tatsächlich »im Alltag " passiert (Pfaff und Kaiser 2006/Schmacke 2007), wenn Heilkundige und Kranke sich begegnen. Forschung und Lehre zum Verständnis der Patientenperspektive ist nun alles andere als neu: man denke an die medizinsoziologischen Arbeiten von Corbin und Strauss (1987, 1988) oder an das Konzept der integrierten Psychosomatik von Uexküll (Adler et al 1993). Es schließt sich am Ende die Frage an, ob die Aufnahme dieses Forschungsfeldes in die "moderne" Agenda der Versor- gungsforschung möglicherweise von der irrigen Annahme ausgeht, Grundlagenforschung sei nicht mehr erforderlich, es gehe gewissermaßen jetzt nur noch um die Implementation gesicherter Forschungsergebnisse in eine erkenntnisresistente Praxis.

\section{Ausgangspunkte der Debatte}

Wenn man sich dem Thema »Patientenperspektive « noch einmal grundlegend nähert, dann wird rasch deutlich, dass erst einmal nicht Wissenschaft, sondern die Gesellschaft das Urheberrecht hat (Francke und Hart 1999, Hart und Francke 2002). Im heutigen Sinne sprechen wir von Patientenperspektive und -interessen, seit Fragen der medizinischen, pflegerischen und psychosozialen Versorgung in Demokratisierungsdebatten, nicht etwa primär in den etablierten Arenen des Gesundheitswesens aufgetaucht sind. Frauengruppen haben die Frage gestellt, warum Schwangerschaft 
fast schon wie eine Krankheit behandelt wird; sie haben hartnäckige Traditionen der Brustkrebschirurgie infrage gestellt; sie haben das gesundheitsgefährdende Konstrukt der Hormon»ersatz «therapie ins Wanken gebracht, wonach der Abfall des Östrogenspiegels nach Einsetzen der Wechseljahre durch langjährige Einnahme von Hormonen »substituiert « werden müsse. Eltern haben sich das Recht erkämpft, bei ihren kranken Kindern im Krankenhaus sein zu dürfen. Juristen und Politiker haben für Patienten das Recht auf Akteneinsicht und umfassende Information wie Aufklärung durchgesetzt.

Begriffe gewinnen leicht eine Eigendynamik, setzen sich im allgemeinen Sprachgebrauch fest und wirken dann selbsterklärend. Die These lautet: Patientenperspektive ist ein solcher moderner Begriff, dessen Verwendung zu garantieren scheint, dass frau oder man sich weit von altmodischen, um nicht zu sagen: vordemokratischen Denkstrukturen befreit hat. Die Anschlussthese lautet: es lohnt sich, bestimmte mit diesem Begriff verbundene Grundannahmen genauer zu untersuchen, um mehr Sicherheit zu der Annahme zu gewinnen, wie groß der Fortschritt bezüglich der Berücksichtigung vitaler Patienteninteressen im Verlauf der letzten Jahrzehnte tatsächlich zu bemessen ist. Eine Annäherung an die Frage, welche Herausforderungen sich für die Forschung bei der Thematik »Patientenperspektive « stellen, wird entlang dreier Begriffspaare versucht.

\section{Paternalismus versus Partnerschaft}

Wer sich über längere Zeiträume für Fragen der Arzt-Patienten-Kommunikation interessiert hat, der reibt sich die Augen, mit welcher Leichtigkeit seit einigen Jahren nahezu inflationär von Shared Decision Making (hölzern mit partizipative Entscheidungsfindung PEF - eingedeutscht) die Rede ist. Ärzte und Ärztinnen entscheiden demnach gemeinsam mit ihren Patienten und Patientinnen über Diagnostik und Therapie. Zwei Kenner der Forschungslandschaft schrieben dazu: "Die Effekte von Empowerment und Shared Decision Making sind bislang weitgehend unklar. Der Ansatz wirkt überhaupt nur bei gut informierten und gebildeten Patienten,

die nicht schwer erkrankt sind « (Grol et al 2005, S. 25, übers. durch den Verf.). Das klingt vielleicht zu pessimistisch, als würden gediegene Forschungstraditionen damit missachtet. Genau das ist aber nicht beabsichtigt. Drei Leuchtturmprojekte sollen genannt werden:

\section{Seit einigen Jahren ist nahezu inflationär von Shared Decision Making die Rede.}

1. Debra Roter and Judith Hall publizieren 1992 »Doctors Talking with Patients - Patients Talking With Doctors«. Wenn ich heute Patienten zuhöre (anekdotische Evidenz), dann wird mir deutlich, wie weit der Weg noch ist, dass Patienten und Patientinnen sich trauen, die ihnen wichtigen Fragen tatsächlich stellen zu können: damit aber müsste der Dialog ja beginnen. Dabei haben Roter und Hall schlüssig belegt, dass es »eigentlich « relativ einfach ist, durch entsprechendes Training in Gesprächsführung und Fallverständnis die Mauer des Schweigens und der unendlichen Missverständnisse in der Arzt-PatientKommunikation zu durchbrechen. Sie beklagten aber in ihrer Bilanz auch, dass das Thema der Arzt-Patient-Beziehung auf einem wünschenswert hohen wissenschaftlichen Niveau für die Praxis keine hohe Priorität habe (Roter und Hall 1992, 173 f.)

2. Gerteis, Edgman-Levitan, Daley und Delbanco publizieren 1993 den Klassiker »Through the Patient's Eyes«. Dieses Buch bilanziert den damaligen Forschungsstand des Picker-Instituts zur Erhebung von Patientenerfahrungen in der Versorgung. Der Sponsor Harvey Picker war durch die Krankheit seiner Frau gemeinsam mit ihr zu der Überzeugung gekommen, dass es notwendig sei, der Patientenperspektive in einem hochtechnisierten und durchaus prinzipiell wertgeschätzten Gesundheitswesen zur gebührenden Anerkennung zu verhelfen. Die aus diesen Ansätzen resultierende »ereignisorientierende Patientenbefragung « ist ein Lehrstück für das Ineinandergreifen von qualitativer und quantitativer versorgungsrelevanter Forschung. Die Nutzung entsprechender Instrumente im klinischen Alltag ist nach wie vor unzureichend. Und vielleicht ist die Aussage der Autorengruppe im Nachwort Ihrer Publikation aktueller als uns lieb ist: »Leistungserbringer und Institutionen im Gesundheitswesen sind bemerkenswert unprofessionell, was die Methoden anbelangt, Rückmeldungen von ihren Patienten zu erhalten « (Gerteis et al 1993, S. 299, Übersetzung durch den Autor).

3. Stewart legt 1995 zusammen mit Brown, Weston et al das Lehrbuch »Patient-Centered Medicine. Transforming the Clinical Method vor. Dort liest man im Zusammenhang mit SDM: »Eine gemeinsame Grundlage zu finden erfordert, dass $\mathrm{Pa}$ tienten und Ärzte wechselseitiges Verständnis und Übereinstimmung über das Wesen der vorhandenen Probleme, über Ziele und Prioritäten der Behandlung und über ihre jeweiligen Rollen in diesen Prozessen erreichen« (S. 96. Übersetzung durch den Autor). Damit sind schwergewichtige Forschungsfelder genannt, die alle eines nahelegen: die prinzipielle Verbesserung der Arzt-Patienten-Kommunikation als unabdingbare Voraussetzung für SDM/PEF ist so notwendig wie schwer zu erreichen. Die gemeinsame Grundlage $\mathrm{zu}$ finden heißt nichts anderes als einen kulturellen Wandel anzumahnen, der jenseits schlichter Implementierungsvorstellungen angesiedelt ist. Wichtig erscheint, dass diese Arbeitsgruppe frühzeitig gezeigt hat, dass eine dezidierte Patientenzentrierung mit besseren Behandlungsergebnissen verbunden sein kann. Und mit Blick auf SDM schreibt Stewart, es wäre ein Missverständnis, Patientenzentrierung bedeute, alle Informationen und Entscheidungen zu teilen: "Patientenzentriert zu sein bedeutet vielmehr, das Bedürfnis von Patienten nach Information und Teilen von Entscheidungen in Betracht zu ziehen und darauf angemessen zu reagieren" (Stewart 2001, S. 445. Übersetzung durch den Autor). Die Notwendigkeit, die Verbesserung der kommunikativen Kompetenzen der Ärzteschaft ins Blickfeld zu nehmen, ist inzwischen weithin anerkannt und wird, wenn auch leider längst noch nicht flächendeckend, in der Medizinerausbildung berücksichtigt. Neben dem 1999 initiierten Reformstudiengang an der Berliner Charité ist hier u. a. der Heidelberger Ansatz hervorzuheben, welche die Anforderungen der neuen ärztlichen Approbationsordnung ernst 
genommen hat. Dort wird - tatsächlich - gefordert, die kommunikative Kompetenz von Studierenden zu fördern, zu trainieren und zu prüfen. Ein derartiges Kommunikations- und Interaktionstraining für Mediziner (Medi-KIT) wurde zunächst in der Inneren Medizin als Pilotprojekt entwickelt. Das Training wurde schrittweise auf zehn klinische Fächer erweitert. Und diese Ausbildung wird mit einem international entwickelten Instrument auf ihren Erfolg hin geprüft: Kommunikation wird damit erstmals ein Prüfungsfach - aus der Überzeugung heraus, dass anders die notwendige Ernsthaftigkeit in der Auseinandersetzung mit dem Thema nicht erreicht werden kann. Und es gibt ermutigende Hinweise, dass dieses Training gegenüber der klassischen Medizinerausbildung nachhaltig die Kommunikationsfertigkeiten im Patientenkontakt verbessert (http://www.medizinischefakultaet-hd.uni-heidelberg.de/MediKIT.108137.0.html).

\section{Gleiche Augenhöhe der Wissensbasis?}

Und ein zweites Mega-Thema lautet: auf welche Informationen können $\mathrm{Pa}$ tienten und Patientinnen sich denn verlassen, wenn sie in den Dialog gehen? Ohne »evidenzbasierte « Informationen ja wohl nicht - also im Grunde auf dieselben Forschungsergebnisse, die Ärzten und Ärztinnen auch zur Verfügung stehen (sollten). "Decision Aids" lautet die Zauberformel, und tatsächlich gibt es inzwischen eine Reihe solcher hochwertiger Entscheidungshilfen, die das Wissen und Verhalten von ratsuchenden Patienten und Patientinnen in erwünschter Weise beeinflussen können: vorausgesetzt, Menschen haben Zugang $\mathrm{zu}$ solchen Materialien, gegebenenfalls Unterstützung durch Dritte, sie besser $\mathrm{zu}$ verstehen, und das passende Zeitfenster, solche Materialien zu nutzen (s. Stacey et al 2011, Légaré et al 2012). Die dahinter liegende Frage aber lautet: wie kann »Literacy« zu Gesundheits- und Krankheitsthemen arzt- wie patientenseitig hergestellt werden? Die bisherigen Erfahrungen zeigen, dass wir bestenfalls am Anfang der Vermittlung der erforderlichen Kulturtechniken stehen (Gaissmair/Gigerenzer G 2008). Es wird in Forschung und Praxis dabei auch noch zu einseitig auf die $\mathrm{Pa}$ - tientenseite geblickt, als sei der dortige Aufklärungsbedarf über medizinische Evidenzen das eigentliche Problem; demgegenüber steht inzwischen außer Frage, dass Ärztinnen und Ärzte ebenso große Probleme haben, mit den inflationär sich vermehrenden Publikationsdaten rational umzugehen (Gigerenzer/Grax 2010).

Es wäre insofern zwar unfair, SDM und PEF als modernistische Kürzel abzutun, weil mit der SDM-Debatte das Thema Patientenzentrierung erneut auf die Tagesordnung gekommen ist. Aber es ist wichtig, sich noch einmal grundlegend mit der Frage zu beschäftigen, wie überhaupt Voraussetzungen für eine Partnerschaft in der ArztPatient-Beziehung geschaffen werden können. Skepsis bleibt angeraten, solange einige schmerzhafte Fragen umschifft werden: Welche Informationsbasis kann arzt- wie patientenseitig zugrunde gelegt werden, wenn von Dialog die Rede ist? In welcher Weise sind beide Seiten trainiert, die vorhandene Datenbasis zu bewerten? In welcher Form werden Arzt- und Patientenperspektive ermittelt und zu einem festen Bestandteil der Kommunikation? Und was bedeutet $» S$ «DM für das professionelle Selbstverständnis und für Patientenerwartungen an die Arztprofession?

Vielleicht geht es nach wie vor eher darum, die Qualität der Kommunikation auf dem Boden von vorbildlichen Ansätzen in Aus-, Fort- und Weiterbildung mit langem Atem zu verbessern als nach den Sternen zu greifen. Schon gar nicht darf es um das Abwälzen von Verantwortung auf Kranke gehen. Auch diese Sorge ist nicht ganz aus der Luft gegriffen. Man könnte überspitzt formulieren: Der mündige Patient wird schnell in Haft genommen (s. Stamer 2012)

\section{Compliance versus Adherence}

Patienten und Patientinnen befolgen die Ratschläge ihrer Ärzte und Ärztinnen oft gar nicht oder auf Dauer nicht - an diesem Problem hat sich über Jahrzehnte die so genannte Compliance-Forschung abgearbeitet. Überwiegend implizit, teils auch explizit wird damit den Kranken die Schuld am Misslingen von Behandlungen und der Vergeudung von Mitteln zugeschoben. Compliance heißt erst einmal schlicht »Folgsamkeit«: Patienten sollen still gehorchen und mehr oder weniger kunstvoll überredet werden. Sozialwissenschaften und Psychologie haben lange vergeblich darum geworben, die Gründe für fehlende Compliance zu verstehen (Petermann und Mühlig 1998). Nun taucht ein neuer und erst einmal etwas spröde klingender Begriff auf: Adhärenz - und damit ist etwas prinzipiell anderes gemeint als Folgsamkeit. Am Beginn der Adhärenz-Debatte steht das Eingeständnis, dass das ewige Klagen über die Non-Compliance von Patientinnen und Patienten nicht weiterführt und dass neue Wege der Verständigung über Ziele und Wege von Behandlung beschritten werden müssen. Einen wichtigen Anstoß zur Adhärenz-Thematik lieferte die Weltgesundheitsorganisation im Jahr 2003 mit ihrem Bericht Adherence to long term therapies: evidence for action. Hier heißt es frei übersetzt: Adhärenz bezeichnet das Ausmaß, in dem das Verhalten einer Person, verordnete Arzneimittel einzunehmen, Ernährungsempfehlungen zu folgen und/oder den eigenen Lebensstil zu ändern, mit wissenschaftlich fundierten Empfehlungen von Behandlern übereinstimmt. Die neue Philosophie lautet: Ärzte und Ärztinnen, Pfleger und Pflegerinnen und alle anderen Fachberufe in der medizinischen, pflegerischen und psychosozialen Versorgung erläutern den Patienten und Patientinnen ihre - fachlich begründete - Sicht der Dinge und erfragen zugleich die Patientensicht zu dem vorgetragenen gesundheitlichen Problem. Im Idealfall entsteht aus dem Dialog eine Vereinbarung über Untersuchungs- und Behandlungsziele, die bei längerem Krankheitsverlauf immer wieder angepasst werden kann. Und dann taucht aufs Neue die spannende Frage auf, wie sehr der Patient oder die Patientin »dabei bleiben ", adhärent sind - und warum sie es gegebenenfalls nicht sind. 


\section{Wie sehen ganz konkrete Bausteine des neuen Adhärenz-Modells aus?}

Es beginnt damit, dass die Therapeuten Interesse daran zeigen, die Meinungen, Haltungen und Wertvorstellungen ihrer Patienten zu erfahren. Dahinter steckt eine weise Einsicht: Ärzte können (und müssen) Experten in Sachen Diagnostik und Therapie sein, aber nur der Kranke kann der Experte seiner Lebensführung sein. Damit ist zweierlei gesagt: professionelles Handeln (und dazu gehören Therapieempfehlungen) bleibt der best vorhandenen Evidenz verpflichtet; die professionellen Helfer sollen nun aber stärker als bisher ihre Aufmerksamkeit auf die Frage lenken, wie sie Patienten dabei unterstützen können, sinnvolle Behandlungsziele in ihren Alltag aufzunehmen.

Es geht weiter damit, sich um Verständlichkeit von medizinischen Botschaften Gedanken zu machen und zu lernen, dass Wissensvermittlung ohne Berücksichtigung der emotionalen Seite der Patientin oder des Patienten oft nicht weiterführt.

Drittens und übergeordnet geht es darum, den Patienten Respekt für ihre Sicht der Dinge zu zollen. Stimmen die ärztliche und patientenseitige Perspektive nicht überein, dann geht es nicht um Überreden oder gar Androhen negativer Konsequenzen, sondern um ein besseres Verstehen, warum Patienten anders denken und ob es Barrieren gibt, die aus dem Wege geräumt werden können. Ist das nicht möglich, dann ist die Entscheidung des Patienten nicht weiter in Frage zu stellen, es sei denn, man müsste jemandem eine (krankheitsbedingte) Einschränkung des Beurteilungsvermögens mit massiver gesundheitlicher Selbstoder Fremdgefährdung unterstellen. Und gleichzeitig bedeutet dies nicht, dass der Arzt oder die Ärztin von ihrer Sicht der Dinge abrücken müssen oder etwa gezwungen werden könnten, gegen ihre Überzeugung zu handeln (Schmacke 2011).

\section{Begründete Non-Compliance und Grundfragen zum Shared Decision Making}

Das alles gilt wohlbemerkt für das Thema »begründete ärztliche Empfehlun- gen«. Adhärenz hat mit anderen Worten - im Gegensatz zu der Anmutung bei »Compliance« - nicht nur etwas mit Unterversorgung zu tun, etwa weil Patientinnen und Patienten nach einem Herzinfarkt nicht die aus ärztlicher Sicht notwendigen Medikamente einnehmen. Es geht auch um Über- und Fehlversorgung, am augenfälligsten bei den zum Teil grotesk anmutenden Mehrfachrezepturen für alte Menschen. Hier kann fehlende »Folgsamkeit « auch nützlich sein, wenn auch das Weglassen verordneter Medikamente eher dem Zufallsprinzip gehorcht. Die Bedeutung des Adhärenz-Konzeptes wird vielleicht noch klarer, wenn man sich vergegenwärtigt, dass es bei akuten wie chronischen Erkrankungen keineswegs immer nur den einen Königsweg der Behandlung gibt. Werden Patienten dann nicht sorgfältig über den jeweiligen Nutzen unterschiedlicher Wege informiert und erfahren Alternativen auf anderen Wegen, kann dies ihre Skepsis gegenüber den erfahrenen Ratschlägen steigern. Und noch einmal ganz grundsätzlich: es ist ja nicht einmal zwingend, dass Therapeuten und Patienten einen tatsächlich vorhandenen Nutzen einer Behandlung gleich bewerten. Was für den einen angemessene Therapie ist, ist für den anderen ein Vorschlag, der Verhaltensänderungen erfordert, die nicht lohnend erscheinen. - Gelegentlich gehen in den Publikationen die Begriffe Adhärenz und Compliance vollkommen durcheinander, oft wird Adhärenz nur

\section{Ein neues Bild des Verhältnisses von Experten zu Laien zeichnet sich erst in Umrissen $a b$.}

gewählt, weil Compliance in Verruf geraten ist, ohne dass der Partizipationsaspekt ernst genommen wird. Wenn Adhärenz aber im Dreiklang von Erfragen der Patientenperspektive, Suche nach Verständlichkeit und emotionaler Akzeptanz wie Respekt vor der Haltung des Patienten verstanden wird, dann ist dieses Konzept immer noch außerordentlich innovativ. Es handelt sich fraglos um relevante Dimensionen für Versorgungsforschung, da ihre Realisierung sich bis heute in den Alltagsroutinen nur lückenhaft auffinden lässt. Schon auf den ersten Blick sind Parallelen zu den
Forschungsfragen erkennbar, welche die Pioniere der SDM-Forschung für das Erkennen und Überwinden von Implementationshürden ihres Konzeptes notiert haben (Légaré F., Ratté S, Karine, Ian G 2008, Légaré, Elwyn, Fishbein et al 2008, Légaré, Stacey, Graham et al 2008). Das Thema ist wiederum nicht etwa vordergründig und ausschließlich »Fehlende Einsicht der Praktiker«. Es ist vielmehr zu konstatieren, dass die theoretische Fundierung des Konstruktes SDM als einer komplexen Intervention unvollständig ist (so auch Scheibler, Janßen, Pfaff 2003). Hierfür soll hier ergänzend der Hinweis genügen, dass Légaré, Stacey, Graham et al bei systematischer Literaturrecherche 161 Definitionen von SDM ausfindig gemacht haben (2008, S. 2). Stamer hat am Beispiel des Umgangs mit den neuen Leitlinien zum kardiovaskulären Gesamtrisiko im hausärztlichen Bereich zudem u. a. gezeigt, dass ein verkürztes Verständnis von SDM im wesentlichen dazu dient, noch mehr Druck auf Patientinnen und Patienten auszuüben, sich »leitliniengerecht« zu verhalten (Stamer 2011).

\section{Das große Forschungsfeld "Experten und Laien"}

Man gewinnt gelegentlich in der Literatur den Eindruck, die Thematisierung von Patientenperspektive folge der Melodie » ̈̈rzte fürchten um ihre tradierte Autonomie - Patienten kämpfen um neue Autonomie«. Es steht für den Autor außer Frage, dass damit tatsächlich ein zentraler Punkt für die unzureichende Wertschätzung der Patientenperspektive in Forschung, Lehre und Praxis getroffen wird. Es soll dennoch zur Klärung der

Forschungsdefizite hier noch einmal argumentiert werden, dass ein neues Bild des Verhältnisses von Experten zu Laien in der medizinischen Versorgung sich erst in Umrissen abzeichnet. Dieser gegentendenzielle, der Political Correctness möglicherweise hinderlich erscheinende Gedanke stellt nun keineswegs in Frage, dass Patienten in der Zivilgesellschaft ein gesetztes Recht auf umfassende Informationen, auf respektvollen Umgang in Klinik und Praxis, auf Unterstützung in Krisen und auf Gehör für ihre Präferenzen haben. Das Problem beginnt ja erst dort, wo diese "Selbstverständlichkeiten" sich an den 
Alltagsroutinen der Versorgung stoßen. Diese offenkundig schwer zu überwindenden Barrieren gilt es besser zu verstehen, wenn von Versorgungsforschung zu Patientenperspektive die Rede ist. Dabei wird erneut deutlich, wie wichtig es ist, sich über die Rahmenbedingungen zu verständigen, die als Autonomie förderlich verstanden werden müssen (s. umfassend bereits Dierks, Bitzer, Lerch et al 2001).

1. Health Literacy ist für Laien eine gewaltige Herausforderung: Zugang zu Wissensbeständen, Verstehen biometrischer Größen und emotionale Stabilität zur Auseinandersetzung mit Forschungsergebnissen und -lücken sind entscheidende Stichworte. Die Experten bleiben zwangsläufig in aller Regel die "Information Broker", auf die es in vielen Situationen ankommt. Internetportale oder Selbsthilfegruppen können diese Probleme nur partiell abmildern, zumal die Vertrauensfrage damit nicht gelöst wird: alles Verstehen ist auf Reduktion von Komplexitäten angewiesen. Die asymmetrische Beziehung zwischen Experten und Laien ist ein fortbestehendes Problem, das nicht mit starken Begriffen wie "gleiche Augenhöhe" oder »Partnerschaft « gelöst werden kann. Auch der »empowerte « Patient bleibt dem Beziehungsgefälle kognitiv und emotional zwangsläufig unterworfen. Und erschwerend kommt hinzu, dass der im Idealfall aufgeklärte Laie nicht darauf rechnen kann, einem aufgeklärten Experten gegenüberzutreten.

2. Health Literacy und Befähigung zur Reflexion der therapeutischen Beziehung stehen vor dem Wagnis von »Shared « Decision Making, ein Unterfangen, das sonst einem enttäuschenden oder gar gefährlichen Blindflug gleicht. Die Kunst des Teilens von Wissen und des Verstehens der Perspektive des anderen müssen eingeübt werden; dies gelingt im klassischen Bildungskanon der Arztprofession bislang offenkundig nicht. Gewähr für die Angemessenheit dieser Aussage ist das gebetsmühlenartig vorgebrachte Argument, man sei ja zu vertiefter Kommunikation bereit, wenn die Zeit dafür da wäre und bezahlt würde.

3. Die Mühen, das Verstehen der Patientenperspektive in das ärztliche Selbstbild aufzunehmen, spiegelt zudem immer noch wider, dass die soziologische und psychologische Forschung zu chronischer Krankheit in der Aus-, Fort- und Weiterbildung der Ärzteschaft nicht die gebührende Rolle spielt. Dies lässt sich an der nach wie vor verkürzten Compliance-Debatte ebenso zeigen wie an dem mühsamen Ringen um ein »Chronic-Care-Modell« vor allem für multimorbide alte Menschen, das sich von dem reflexartigen Eingehen auf die Symptomatologie der Patienten löst und stattdessen versucht, die Priorisierung der Versorgungsprobleme zur Leitidee zu nehmen und zugleich danach zu fragen, welchen Beitrag die verschiedenen Fachberufe dabei leisten können. Stattdessen sind chronisch Kranke häufig nicht nur mit der Krankheitsarbeit an sich belastet sondern auch mit der Organisation ihrer Versorgung: eine wenig beachtete Verkehrung »traditioneller « Rollen.

4. Von der gedanklichen Hereinnahme der Patientenperspektive in die Debatten bis hin zu einer echten Partnerschaft bleibt ein weiter Weg. Man muss vielleicht auch die Frage zulassen, ob das Konstrukt »Partnerschaft « nicht ein unzureichender Versuch der Glättung ungleicher Rollen ist, im schlimmsten Fall sogar ein gefährlicher Versuch, wenn nämlich der Patient entweder zum umworbenen Kunden umfunktioniert wird oder in eine Verantwortung gestellt wird, die er nicht ertragen kann. Erst einmal bleibt nüchtern zu sehen, so die These, dass Ärzte und Ärztinnen einem »Eigen-Sinn « folgen, der mit dem »Eigen-Sinn« der Patienten und Patientinnen gar nicht identisch sein kann (Richter/Stamer/Schmacke 2012, Schmacke/ Stamer/Richter 2011). Professionalität bleibt unteilbar. Sie ist allerdings erst dann des Vertrauens wert, wenn sie die Befähigung zum Perspektivwechsel beinhaltet und Raum für die Verständigung über unterschiedliche Perspektiven bietet.

5. Über Profession, Professionalität und Selbstbilder genauer nachzudenken, scheint auch noch aus einer anderen Betrachtung heraus lohnend zu sein. In einer frühen Arbeit zum Themenkreis Qualitätssicherung in der Medizin hat Blumenthal (1996) darauf aufmerksam gemacht, dass die Ärzteschaft sich vermutlich auch deshalb mit der Kritik an der Qualität der Medizin so schwer tue, weil diese Kritik sie in einer historischen Phase ungekannter Höchstleistungen der kurativen Medizin erreichte und als ungerechte Schelte der Gesellschaft missverstanden wurde - und wird. Damit stehen Außenkritik und Binnenwahrnehmung in einem scheinbar paradoxen Verhältnis zueinander. Es geht aber vermutlich genau darum, dass die Arztprofession die Einbindung der $\mathrm{Pa}$ tientenperspektive als identitätsstiftend und nicht als von außen erzwungen erlebt. Dies könnte der tiefer liegende Grund dafür sein, dass die heute übliche Brandmarkung der paternalistischen Medizin nur scheinbar schon den Weg zu einem anderen Verständnis der Arzt-Patienten-Beziehung signalisiert. Patienten und Patientinnen sollten jedenfalls davor geschützt werden, mit einem unreflektierten Verständnis von »Shared « Decision Making konfrontiert zu werden, das sie weitaus hilfloser zurücklassen kann als ein sich explizit anwaltschaftlich verstehender benevolenter Paternalismus dies je vermöchte.

Inwieweit professionelle Sicht, Patientenpräferenzen und evidenzbasierte Medizin tatsächlich überein zu bringen sind (Schmacke 2006), ist eine herausfordernde Frage an die Versorgungsforschung. Sie ist sicher nicht trivial, ihre Beantwortung bedarf aber nicht der Erfindung neuer Methoden, wenn man auf dem Stand zur Entwicklung und Evaluation komplexer Interventionen aufsetzt (Schmacke 2007). In die Vorüberlegungen derartiger Studiendesigns muss vor allem einfließen, welche bereits angemessen untersuchten Elemente einer patientenzentrierten Medizin in einer komplexen Intervention zusammen gebunden und gegen »Regelversorgung « getestet werden sollen (Légaré et al 2010). Das ist in weiten Teilen "HardCore-Grundlagenforschung «. Man könnte insofern als Paradoxon und Vision formulieren: Versorgungsforschung brauchen wir eines Tages nicht mehr, wenn versorgungsrelevante Forschungsfragen und -perspektiven Teil der klinischen Forschung geworden sind. Das könnte allerdings etwas dauern.

Das Literaturverzeichnis finden Sie unter www.gus.nomos.de 